\title{
Adaptive methods help drug sponsors find best treatment dose
}

Across the drug industry, around $20 \%$ of clinical trials now involve some element of adaptive design, in which researchers make preplanned adjustments to protocols at interim checkpoints. The idea is to save both time and money, helping drug developers collect data and modify parameters midstudy. But while adaptive trials have commonly involved simple changes-mostly futility analyses or sample size reestimations-a new effort is underway to facilitate more sophisticated adjustments to drug dosages in these types of clinical studies.

On 18 February, three large pharmaceutical companies and a contract research organization (CRO) specializing in adaptive trial design announced a new coalition with this aim. The ADDPLAN DF Consortium so far includes Novartis, Janssen Pharmaceuticals and Eli Lilly. Four other major drugmakers are also in discussions about joining.

"This announcement is a major step forward," says Ken Getz of the Tufts Center for the Study of Drug Development in Boston. "It's widely expected that adaptive dose finding - a very promising approach that few companies are using routinely at this time-has the potential to improve the success rates of drugs in later-stage development programs."

Adaptive dose-finding trials are starting to be put to the test. Last year, for example, physicians at ten US medical centers launched a 250-person trial designed to evaluate whether infusions of the amino acid derivative $\mathrm{L}$-carnitine can reduce organ failure in patients with septic shock. Ordinarily, the study's organizers would have to define the ratio of participants in each treatment arm from the outset of the trial. Instead, at scheduled points in the trial-every 12 participants following a 40-person 'burn-in' phase-a monitoring committee will peek at the interim data and then preferentially allocate participants to the $\mathrm{L}$-carnitine dose that is proving the most effective (although a fixed proportion of the participants will always receive placebo).

"You're changing the arms they're randomized to based on almost real-time information," says principal investigator Alan Jones, research director of the emergency medicine department at the University of Mississippi Medical Center in Jackson, who described the study methodology last year in the journal Critical Care Medicine (41, $1674-1678,2013)$. If the best dose is also

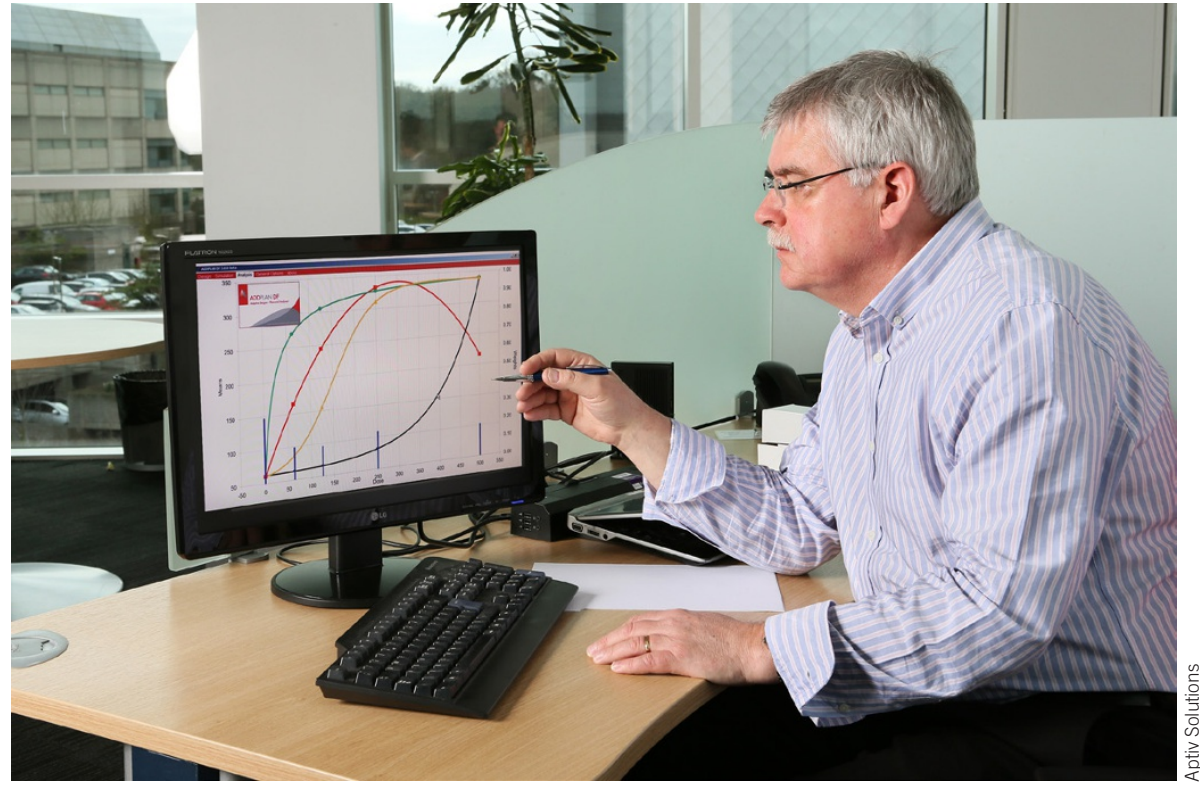

Ahead of the curve: Andy Grieve examines dose-response shapes from a simulated phase 2 trial.

saving more lives, the trial will be stopped early so that the study sponsors can move swiftly ahead to test this optimal dose in a larger phase 3 trial.

\section{Pivotal planning}

The issue of picking the best drug dose in the exploratory stages of clinical development is no trivial matter. "If you get the dose selection right in phase 2 , then you're reducing the chances of failure in phase 3," says Andy Grieve, senior vice-president of clinical trials methodology at Aptiv Solutions, the Virginia-headquartered CRO anchoring the new ADDPLAN DF Consortium. Currently, around half of all pivotal phase 3 trials fail to meet their primary efficacy endpoints. "I would suggest that $70-80 \%$ of that is getting the dose selection wrong," Grieve says.

The ADDPLAN DF Consortium is so named because it revolves around a software platform developed for the design, planning and analysis of dose-finding trials. ADDPLAN DF, released commercially by Aptiv last year, uses a method called multiple comparison procedure modeling, or MCPMod. Instead of comparing experimental doses head to head with placebo as most traditional statistical analyses do, MCPMod compares different doses to each other (as well as to placebo); it then fits the data to a handful of the most plausible doseresponse relationships to help inform which doses should advance into phase 3 testing.
The European Medicines Agency (EMA) endorsed the method earlier this year, and discussions are ongoing with the US Food and Drug Administration about validating the tool, as well.

MCP-Mod has the advantage that it allows investigators to include a wider range of drug doses without requiring larger sample sizes. But as written, MCP-Mod does not permit sponsors to use interim analyses to alter trial dynamics in progress. That should soon change: the new ADDPLAN DF Consortium is planning to produce an adaptive version of the software that can inform ongoing dose allotment and subject randomization by year's end. Because the basic statistical approach would be the same, this adaptive update would not need to be separately qualified or validated by drug regulators.

Christina Yap, a biostatistician and study methodologist at the University of Birmingham, UK, sees developments like the ADDPLAN DF Consortium as part of a larger trend in adaptive dose finding. "It's only in recent years that there's been great interest in the implementation of such novel designs," says Yap, who helped develop the protocol behind a trial launched earlier this year in people with acute myeloid leukemia-the UK's first such adaptive dose-finding study in hematology to date. "People are realizing that early-stage trials are really important to do right."

Elie Dolgin 